\title{
FUENTES PROTEICAS DE BAJA DEGRADACIÓN RUMINAL Y SU EFECTO EN LA PRODUCCIÓN DE METANO EN BOVINOS DE CARNE
}

\author{
Protein sources of low ruminal degradation and it effect on methane \\ production in beef cattle
}

\section{Artículo de Revisión}

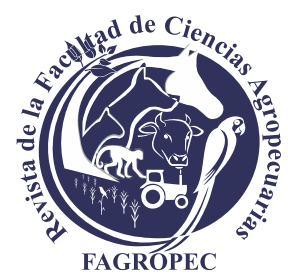

Recibido 20 de julio de 2020

Aceptado 10 de noviembre 2020

'Zoot. Departamento de Zootecnia, Facultad de Ciencias Agrarias y Veterinarias (FCAV), Universidade Estadual Paulista "Júlio de Mesquita Filho"(UNESP), Jaboticabal, São Paulo.

E-mail: thayna17.rp@outlook.com

${ }^{2} \mathrm{PhD}$, Msc, MVZ. Investigador $\mathrm{PhD}$ en Nutrición de Rumiantes, AGROSAVIA, San Roque, Antioquia. E-mail: yurygranjasalcedo@gmail.com

D https://orcid.org/0000-0003-4783-999X

${ }^{3}$ Vet. Facultad de Ciencias Exactas, Naturales y Agropecuarias de la Universidad de Santander.

E-mail:danielle2205av@gmail.com

https://orcid.org/0000-0002-9158-7155

${ }^{4} \mathrm{PhD}$, Msc, Zoot. Departamento de Zootecnia, Facultad de Ciencias Agrarias y Veterinarias (FCAV), Universidade Estadual Paulista "Júlio de Mesquita Filho" (UNESP), Jaboticabal, São Paulo.

E-mail:_uarte_juliana@hotmail.com

(iD https://orcid.org/0000-0001-7462-9010

\section{Cómo citar}

Ribeiro da Silva, T., Granja-Salcedo, Y. T., Alvarado-Vesga, D., \& Duarte-Messana, J. (2020). Fuentes proteicas de baja degradación ruminal y su efecto en la producción de metano en bovinos de carne. Revista Facultad de Ciencias Agropecuarias -FAGROPEC. Universidad de la Amazonia. Vol. 12 (2), 232240. https://doi.org/10.47847/fagropec.v12n2a5

*Autor para correspondencia: yurygranjasalcedo@gmail.com Licencia Creative Commons (CC BY 4.0)

\author{
Thayná Ribeiro da Silva ${ }^{1}$ \\ Yury Tatiana Granja Salcedo ${ }^{2 *}$ \\ Daniela Alvarado Vesga ${ }^{3}$ \\ Juliana Duarte Messana ${ }^{4}$
}

\section{RESUMEN}

La producción pecuaria ha sido estigmatizada en función de la producción de gases efecto invernadero (GEI), principalmente de gas metano $\left(\mathrm{CH}_{4}\right)$. Sin embargo, la mayoría de la población desconoce las cifras reales que aporta cada sector económico a la producción de estos gases en el mundo. La Organización de las Naciones Unidas (ONU) estima que la población mundial llegará a ser de 8,6 billones en 2030 y de 9,8 billones en 2050; consecuentemente habrá necesidad de aumentar la producción de alimentos para el consumo humano y consecuentemente animal. Dentro de las estrategias de mitigación de producción de $\mathrm{CH}_{4}$, la manipulación de las dietas se concibe como un enfoque simple y práctico, que puede garantizar una mejor productividad, además de ayudar a reducir las emisiones de $\mathrm{CH}_{4}$ hasta en un $40 \%$. Es posible clasificar la proteína de la dieta con respecto al lugar de aprovechamiento por el animal, es decir, puede ser denominada como proteína degradada en el rumen (PDR) o proteína no degradable en el rumen (PNDR). Por otro lado, se conoce que a partir de la fermentación ruminal de carbohidratos y de proteínas ocurre la producción de $\mathrm{CH}_{4}$ entérico por los bovinos, así, el uso de $\mathrm{PNDR}$ podría limitar el crecimiento de los microorganismos metanogenicos debido a la baja disponibilidad de grupos $\mathrm{H}_{2}$ y metilo en el rumen para su metabolismo, reduciendo consecuentemente las emisiones de $\mathrm{Ch}_{4}$. El objetivo de esta revisión se basó en exponer los detalles del metabolismo ruminal y resultados de estudios recientes, utilizando fuentes de PNDR para reducción de las emisiones de $\mathrm{CH}_{4}$ en rumiantes.

Palabras clave

Archaeas; confinamiento; fermentación; ganadería; pasto.

\section{ABSTRACT}

Livestock production has been stigmatized based on the production of greenhouse gases (GHG), mainly methane gas $\left(\mathrm{CH}_{4}\right)$. However, most of the population does not know the real numbers of the contribution of each economic sector to the production of these gases all over world. The United Nations (UN) estimates that the world population will reach 8.6 billion in 2030 and 9.8 billion in 2050, bringing the need to increase the production of food for humans and consequently animal consumption. Within the $\mathrm{CH}_{4}$ production mitigation strategies, the dietary manipulation is a simple and practical approach, which can guarantee better productivity, as well as reducing $\mathrm{CH}_{4}$ 
REVISTA FAGROPEC

UNIVERSIDAD DE LA AMAZONIA - FLORENCIA, CAQUETÁ

Fuentes proteicas de baja degradación ruminal y su efecto en la producción

de metano en bovinos de carne

emissions by up to $40 \%$. There is a possibility to classify dietary protein in relation to the place of the animal use, that is, it can be called rumen degraded protein (RDP) or rumen undegradable protein (RUP). On the other hand, it is known that the ruminal fermentation of carbohydrates and proteins lead to the cattle's production of enteric $\mathrm{CH}_{4}$. In such a way, the use of RUP could limit the growth of methanogenic microorganisms due to the low availability of $\mathrm{H}_{2}$ and methyl groups in the rumen for its metabolism, consequently reducing the $\mathrm{CH}_{4}$ emissions. This review aims to present details of ruminal metabolism and the results of recent studies using RUP sources to reduce $\mathrm{CH}_{4}$ emissions in ruminants.

Key words

Archaeas; feedlot; fermentation; forage; livestock.

\section{INTRODUCCIÓN}

La Organización de las Naciones Unidas (ONU) estima que la población mundial llegará a ser de 8,6 billones en 2030 y de 9,8 billones en 2050 (FAO, 2019), por lo que será necesario aumentar la producción de alimentos para el consumo humano y consecuentemente animal. En este contexto, la ganadería de carne deberá ser cada vez más eficiente siendo necesario producir carne de calidad a bajo costo y con el menor impacto ambiental posible. Una alternativa para lograr lo antes mencionado es intensificar el sistema de producción, con el fin de atender esa creciente demanda implementando la ceba en confinamiento, la cual es una opción que mejora los índices de productividad así como la calidad de carne.

En el entendido de que todos los países de América Latina son productores de carne, se deberán tomar medidas tecnológicas para el aumento de los índices productivos que sean necesarios. Por ejemplo, en los últimos años Brasil se ha destacado en el mercado como un importante productor de alimentos por su ganadería de carne, actuando competitivamente en el mercado internacional; de acuerdo con USDA (2020), este país posee el mayor rebaño comercial de bovinos en el mundo, pues cuenta con 238,1 millones de cabezas y es el segundo país más consumidor de carne (37,5 kg/habitante/año), además de ser un líder mundial en la exportación de carne bovina. Así mismo, en Brasil el sector de la ganadería representa un importante papel en el escenario nacional, en tanto contribuye con aproximadamente el 8\% del PIB del país, lo cual refleja un estructurado proceso de desarrollo, el cual permitió a su vez no solo el aumento de la productividad, sino también de la calidad del producto, así como una mayor competitividad y cobertura del mercado. Por otro lado, los sistemas ganaderos en Colombia están principalmente enfocados a la producción de carne; menos del 1\% está dedicado a la lechería especializada; el 21,1\% de ellos están dedicados a la ceba; el 41,4\% a la cría (parte de la cadena productiva de carne); finalmente el restante al doble propósito (FEDEGAN, 2018).

El objetivo de esta revisión se basa en exponer los detalles del metabolismo ruminal y resultados de estudios recientes utilizando fuentes de proteína no degradable en el rumen (PNDR), para reducción de las emisiones de metano entérico $\left(\mathrm{CH}_{4}\right)$ en bovinos de carne.

\section{Impacto ambiental de la ganadería y fermentación ruminal}

En función de las proyecciones realizadas por la FAO se espera que el consumo de proteína animal debe aumentar, debido tanto al crecimiento de la población, como al aumento de la renta per cápita, especialmente en los países en desarrollo, hecho que conlleva la búsqueda de alimentos de calidad con valor agregado. En este contexto, mucho se ha estigmatizado a la producción pecuaria en función de la producción de gases de efecto invernadero (GEI), principalmente de gas metano $\left(\mathrm{CH}_{4}\right)$. Sin embargo, la mayoría de la población desconoce las cifras reales del aporte de cada sector económico a la producción de estos gases en el mundo. 
Anivel mundial, dentro de los sistemas de producción de carne, además de la búsqueda de la intensificación de dicho sistema, existe una preocupación por la producción animal de forma sostenible, con el objetivo de reducir los impactos ambientales y los cambios climáticos globales. De acuerdo con el informe del Intergovernmental Panel on Climate Change (IPCC, 2019), en América Latina en promedio, un bovino emite $56 \mathrm{~kg} /$ animal $/$ año de metano entérico, mientras que la actividad agropecuaria fue responsable del $23 \%$ del total de emisiones antropogénicas de gases de efecto invernadero (GEI) y del $44 \%$ de las emisiones de metano $\left(\mathrm{CH}_{4}\right)$, entre los años 2007 y 2016 en el mundo. Por lo tanto, será necesario producir más alimentos y al mismo tiempo conservar los recursos naturales disponibles, reduciendo las emisiones de GEI.

En este contexto, los rumiantes poseen un papel importante tanto para la producción de alimentos, como para la contribución de las emisiones de GEI. Estos han evolucionado durante más de 14 millones de años, para adaptarse a diferentes regiones del mundo y para aprovechar los alimentos de alta disponibilidad en la naturaleza (plantas) (Van Soest, 1994). Dicha evolución les ha permitido mantener una microbiota simbiótica diversa en su rumen, constituida principalmente de bacterias, Archaeas, protozoarios, hongos y virus (Firkins \& Yu, 2015), lo que permite a los rumiantes la capacidad de degradar fibras vegetales y aprovechar el nitrógeno no proteico (NNP), como la urea, los nitritos y los nitratos. Sin esta simbiosis, los componentes fibrosos no podrían ser aprovechados por el animal, una vez la mayoría de los mamíferos no tienen la capacidad de producir enzimas que rompan los enlaces $\beta 1-4$, que une a la mayoría de las hexosas que componen la celulosa y la hemicelulosa (Van Soest, 1994). De esta población, las bacterias son el grupo más estudiado, en bovinos a pasto esta población es representada principalmente por bacterias de los filos Firmicutes, Bacteriodetes, Proteobacteria y Fibrobacteres (Granja-Salcedo et al., 2017).

Estudios sugieren que la diversidad de los microorganismos ruminales está asociada con características productivas del rumiantes hospedero, así como con la sanidad del ganado que influye en la eficiencia alimentaria (Li \& Guan, 2017; Shabat etal., 2016) y en la producción de $\mathrm{CH}_{4}$ (Difford etal., 2018; Wallace etal., 2015). Siendo así, los bovinos que presenten una mayor eficiencia en la digestión de alimentos asociado a una dieta que contribuya con la reducción de $\mathrm{CH}_{4}$ entérico, puede beneficiar la producción de rumiantes y mejorar la rentabilidad y la sostenibilidad, con un menor impacto ambiental.

De acuerdo conHaque (2018), dentro de las estrategias de mitigación de producción de $\mathrm{CH}_{4}$, la manipulación de las dietas es un enfoque simple y practico, que puede garantizar una mejor productividad, además de reducir las emisiones de $\mathrm{CH}_{4}$ hasta en un $40 \%$. Una de las estrategias nutricionales propuestas para reducir la producción de $\mathrm{CH}_{4}$ es aumentar la inclusión de nutrientes potencialmente digestibles, en dietas que escapen a la fermentación ruminal, o también el uso de dietas con perfiles de fermentación que favorezcan la formación de ácido propionico en vez de ácido acético o butírico (Beauchemin et al., 2008; Dijkstra etal., 2011; Ellis et al., 2008). En este sentido, un estudio en bovinos de la raza Nelore demostró que el aumento de carbohidratos no estructurales en la dieta (almidón), puede reducir hasta 50\% de la población de Arqueas metanógenas en el rumen de estos animales y consecuentemente disminuir de 57,0 para 49, 5 gr de $\mathrm{CH}_{4}$ producido por cada $\mathrm{kg} \mathrm{de}$ alimento consumido por el animal (Granja-Salcedo et al, 2016).

Con base en la ruta de fermentación, la formación de acetato (C3) y butirato (C4) provienen de la fermentación de carbohidratos estructurales que resultan en la liberación de $\mathrm{H}_{2} \mathrm{y}$ carbono en la forma de $\mathrm{CO}_{2}$, y por lo tanto, en la formación de $\mathrm{CH}_{4}$, mientras que el propionato (C3) es producido principalmente durante la fermentación de carbohidratos no estructurales, no libera $\mathrm{CO} 2$ y consume $\mathrm{H}_{2}$, reduciendo así la producción de $\mathrm{Ch}_{4}($ Dijkstra etal., 2011; Ellis etal., 2008).

A partir de la fermentación entérica, los rumiantes son capaces de transformar la biomasa no digerible por lo humanos (ejemplo: celulosa y hemicelulosa) en proteína animal de excelente calidad (Gerber et al., 2015). Con respecto a la cantidad de $\mathrm{CH}_{4}$ producido, este depende de la cantidad de biomasa degradada, la fracción de 
biomasa no incorporada por los microorganismos, el ácido graso formado durante la fermentación ruminal, el tipo de substrato fermentado y la composición de la dieta (Bannink et al., 2010; Dijkstra et al., 2011; van Gastelen et al., 2019).

Durante la fermentación, el hidrógeno $\left(\mathrm{H}_{2}\right)$ y el gas carbónico $\left(\mathrm{CO}_{2}\right)$ son liberados en el rumen a través de la reoxidación de los cofactores reducidos (NADH, NADPH y FADH). Estos son los principales sustratos utilizados por los microorganismos metanogénicos (Archaeas) en la vía predominante de producción de $\mathrm{CH}_{4}$ en el rumen, conllevando a la reducción de $\mathrm{H}_{2} \mathrm{y}$ proporcionando una fermentación continua(Ellis et al., 2008). Esto ocurre para garantizar un ambiente ideal para la fermentación realizada por los microorganismos, caracterizando un camino crucial para mantener el equilibrio del $\mathrm{H}_{2}$ y elpH ruminal, una vez acúmulos de $\mathrm{H}_{2}$ resultan en bajo $\mathrm{pH}$ y baja disponibilidad de los cofactores (NAD, NADP y FAD), reduciendo así la actividad de diversas enzimas microbianas importantes para la continuidad de la degradación de alimentos en el rumen.

\section{Proteína en la dieta y su efecto en la producción de metano entérico}

Adicionalmente, la fermentación de proteína en el rumen hace que los aminoácidos (AAs) estén disponibles para la desaminación, resultando en una mayor producción de $\mathrm{H}_{2}$ (Vanegas et al., 2017). Según Salazar et al. (2008) el metabolismo de proteína en rumiantes envuelve la degradación de proteína en péptidos, AAs y amoniaco $\left(\mathrm{NH}_{3}\right)$ por los microorganismos ruminales. La estructura de la proteína en la dieta es considerada un factor determinante de ese metabolismo, influenciando la degradación ruminal(Bach et al., 2005).

Un estudio realizado por Vanegas etal., (2017) analizó la influencia de la fuente de nitrógeno (N) en la producción de $\mathrm{CH}_{4}$ in vitro a partir de la fermentación de dos sustratos, preparados a partir de una mezcla de almidón de maíz y celulosa en diferentes proporciones, con tasas variables de fermentación y observó que diferentes substratos influencian la disponibilidad de grupos metil e $\mathrm{H}_{2}$, afectando la actividad de los microorganismos metanogénicos, alterando las poblaciones microbianas y generando diferentes producciones de $\mathrm{CH}_{4}$.

Adicionalmente, Haro et al., (2018) estudió el efecto de la inclusión de proteína protegida en la degradación ruminal sobre la producción de $\mathrm{CH}_{4}$ y fermentación ruminal in vitro, confirmando que el uso de proteína promueve una menor producción de $\mathrm{CH}_{4}$ debido a la menor degradación de proteínas en el rumen, además de aumentar la producción de propionato. Bento et al. (2015) observaron que la suplementación con caseína, una fuente de PDR, infundida directamente en el rumen de novillos Nelore, provocó un aumento en la actividad de desaminación de aminoácidos, hecho que es positivo para la cantidad de sustrato disponible para la microbiota ruminal y para el rumiante. Sin embargo, no observaron efectos de los tratamientos sobre la diversidad de Archaeas metanogénicas.

En este contexto, es posible clasificar la proteína de la dieta con respecto al lugar de aprovechamiento por el animal, es decir, puede ser denominada como proteína degradada en el rumen (PDR) o proteína no degradable en el rumen (PNDR). La PDR es compuesta de NNP y proteína verdadera, siendo esta última degradada en péptidos y AAs, que serán desaminados y utilizados para la síntesis de proteína microbiana o siendo degradados por los microorganismos ruminales. Por otro lado, la PNDR se caracteriza por tener una baja degradación ruminal, encontrandose disponible en mayor cantidad en la digestión post ruminal, es decir en el intestino delgado.

Dietas que proporcionan PDR en grandes cantidades pueden ocasionar exceso de $\mathrm{NH}_{3}$, el cual será absorbido por la pared ruminal y enviado al hígado, donde será transformado en urea; sin embargo, puede retornar al rumen a través de la saliva o ser excretado vía orina (Salazar et al., 2008). Una vez en el ambiente, el N excretado puede ser convertido en óxido nitroso y $\mathrm{CO}_{2}$, siendo perjudiciales para la salud humana y para el medio ambiente (Abbasi et al., 2018). La eficiencia en la utilización de nitrógeno por los bovinos es aproximadamente del $30 \%$ y el otro $70 \%$ es excretado por el animal al medio ambiente, por medio de las heces 
y de la orina(VandeHaar y St-Pierre, 2006).

El uso de PDR está asociado con el suministro de proteína microbiana en el intestino delgado, el cual satisface parte de la demanda de proteínas metabolizables del animal. Sin embargo, la adición de PNDR en la dieta puede intensificar el flujo de AAs para el intestino delgado y mantener así el equilibrio de la producción (Detmann et al., 2014; Leonardi et al., 2003; Rufino et al., 2016); por lo anterior, una mayor cantidad de proteína absorbida en el intestino delgado, aumenta la retención de $\mathrm{N}$ en el cuerpo del animal.

De esta forma, se conoce que a partir de la fermentación ruminal de carbohidratos y proteínas ocurre la producción de $\mathrm{CH}_{4}$ entérico por los bovinos. La producción de $\mathrm{CH}_{4}$ es realizada exclusivamente por las Archaeas metanogénicas, sin embargo, todos los microorganismos ruminales participan de este proceso directa o indirectamente(Yang et al., 2016).

Las Archaeas son un grupo de microorganismos primitivos que se diferencian de las bacterias. Estos microorganismos habitan ambientes extremos y son en su mayoría anaeróbicos, por lo cual viven en ambientes con pocas concentraciones de oxígeno. Su pared celular es compuesta por pseudomureina, proteína, glicoproteína o heteropolisacáridos y la secuencia de nucleótidos indica que presentan una evolución inicial diferentes a la de las bacterias(Ishino et al., 1998).

En el ambiente ruminal, una pequeña parte de las metanogénicas se encuentra asociada a los protozoarios, tanto en su superficie como en su interior y en su mayoría están de forma libre en el líquido ruminal o como miembros de la biopelícula adheridos a las partículas de alimento (Valle et al., 2015).

La asociación entre los metanogénicos y los demás microorganismos en el rumen revela una relación simbiótica, en la cual los metanogénicos, al utilizar el $\mathrm{H}_{2}$, promueve un ambiente ruminal adecuado para la supervivencia de estas (Van Soest, 1994).

Sin embargo, también se reporta una relación competitiva entre los microorganismos metanogénicos, las bacterias reductoras de sulfato y acetogénicas, debido a la disponibilidad de $\mathrm{H}_{2}$ en el rumen (Ellis et al., 2008). Por otra parte, otros compuestos como formato, metilaminas y sulfuros metilados tambien pueden ser usados como sustratos, por los microorganismos metanogenicos.

Por lo anterior, el uso de PNDR podría limitar el crecimiento de los microorganismos metanogénicos, debido a la baja disponibilidad de grupos $\mathrm{H}_{2}$ y metilo en el rumen para su metabolismo, reduciendo consecuentemente las emisiones de $\mathrm{CH}_{4}$. Estudios recientes realizados en Brasil por Da Silva (2020) sobre novillos Nelore, los cuales han sido mantenidos en confinamiento y con diferentes fuentes de PNDR en la dieta (gluten de maíz o harina de soja sobrepasante), lograron demostrar que estas fuentes de PNDR reducen entre 70 y $75 \%$ la población total de arqueas productoras de metano en el rumen de estos animales, al compararse con un grupo control con harina de soja como fuente de PDR. Una evaluación similar, pero en animales mantenidos en pastos Urochloa brizantha cv. Xaraés (Braquiária brizantha Xaraés) suplementados con concentrados con las mismas fuentes de PNDR, Colovate (2020), encontró reducciones entre 69 y $72 \%$ de la población total de arqueas productoras de metano en el rumen de novillos Nelore. Sin embargo, cuando estos trabajos midieron las emisiones de $\mathrm{CH}_{4}$ en los animales no encontraron efectos de reducción, al compararse con las observadas en animales de la dieta control. Estos estudios están en línea con afirmaciones realizadas por Tapio et al., (2017), quienes sugieren que las emisiones de metano entérico en rumiantes dependen más de la diversidad de la comunidad de arqueas en el rumen y no tanto de la abundancia de esta población en ese ambiente. Así, etapas de experimentación futuras son necesarias para estudiar a profundidad la diversidad microbiana ruminal de esta población, en animales alimentados con fuentes de PNDR. Así mismo, es necesario realizar estudios económicos en los cuales, factores como la ganancia de peso y el tiempo de producción de estos animales sea considerado. 


\section{CONCLUSIONES}

La manipulación dietética para el incremento de los índices productivos de los sistemas de producción de carne en Latinoamérica, debe ser considerada una estrategia importante respecto del objetivo de lograr sistemas ganaderos sustentables, que produzcan carne de calidad, pero con el menor impacto ambiental posible. El uso de fuentes de proteína no degradable en el rumen puede ser una alternativa a considerar para alcanzar este objetivo; sin embargo, debido a los pocos resultados experimentales, futuras investigaciones deben ser conducidas, con el fin de comprender mejor sus efectos sobre la población de Archaeas, además de los posibles beneficios ambientales.

\section{LITERATURA CITADA}

Abbasi-raja, I., Abbasi, F., El Hack-Abd, M., Mervat- Latif, A., Rab, S., Hayat, K., Mohamed, A., Bello, B., Junhu, Y., Yangchun, C,. (2017). Critical analysis of excessive utilization of crude protein in ruminants ration: Impact on environmental ecosystem and opportunities of supplementation of limiting amino acids - a review. Environmental Science and Pollution Research, Environ Sci Pollut Res. Northwest A\&F University, China 25(1), 181-190. https://doi.org/10.1007/s11356-017-0555-4

Bach, A., Calsamiglia, S., Stern, M. (2004). Nitrogen Metabolism in the Rumen. American Dairy Science Association, J. Dairy Sci. Institucio' Catalana de Recerca i Estudis Avanc, ats, Barcelona-España. 88, E9-E21.https://doi.org/10.3168/jds.S0022-0302(05)73133-7

Bannink, A., Smits, M., Kebreab, E., Mills; Ellis, J., Klop, A., France, J., Dijkstra, J. (2009). Simulating the effects of grassland management and grass ensiling on methane emission from lactating cows. The Journal of Agricultural Science, J. Agri Sci. Department of Animal Production, Animal Sciences Group. 148(1), 55-72. https://doi.org/10.1017/S0021859609990499

Beauchemin, K., Kreuzer, M., O'mara, F., y Mcallister, T.(2008). Nutritional management for enteric methane abatement: A review. Australian Journal of Experimental Agriculture, Aust J ExAgri. Agriculture and Agri-Food Canada, Research Centre, Lethbridge, Alberta, Canada. 48(2), 21-27. https://doi.org/10.1071/EA07199

Bento-Pereira, C., Azevedo, A., Gomes, D., Batista, ED., Rufino-Almeida, M., Detmann, E., Mantovani, H. (2015). Effect of protein supplementation on ruminal parameters and microbial community fingerprint of Nellore steers fed tropical forages. Animal. Departamento de Microbiologia, Universidade Federal de Viçosa, Minas Gerais, Brazil; v.10, p. 44-54. https://doi.org/10.1017/S1751731115001512

Colovate, PH. (2020). Influência de diferentes fontes de proteína não degradável no rúmen sobre Archaeas metanogênicas e emissão de metano entérico em bovinos Nelore terminados em pastagens. Tesis conducente al grado de Zootecnia. Directora: Dra. Telma Teresinha Berchielli. Universidade Estadual Paulista, Campus Jaboticabal. Carrera de Zooctenia. 42p.

Da Silva, TR. (2020). Influência de diferentes fontes de PNDR sobre a emissão de metano entérico e Archaeas metanogênicas em bovinos Nelore confinados. Tesis conducente al grado de Zootecnia. Directora: Dra. Telma Teresinha Berchielli. Universidade Estadual Paulista, Campus Jaboticabal. Carrera de Zooctenia. $37 \mathrm{p}$

Detmann, E., Valente, E., Batista, ED.; Huhtanen, P., (2014). An evaluation of the performance and efficiency of nitrogen utilization in cattle fed tropical grass pastures with supplementation. Livestock Science, J Liv 
Sci. Universidade Federal de Viçosa, Minas Gerais - Brasil. 162, 141-153. https://bit.ly/39AbA0i

Difford, GF., Plichta, RD., Løvendahl, P., Lassen, J., Joan-Noel, S., Højberg, AO., Wright, D., Zhu, Z., Kristensen, L., Bjørn-Nielsen, H., Guldbrandtsen, B., Sahana, G. (2018). Host genetics and the rumen microbiome jointly associate with methane emissions in dairy cows. PLOS Genetics, Aarhus University, Tjele-Denmark. 14(10): e1007580. https://doi.org/10.1371/journal.pgen.1007580

Dijkstra, J., Oenema, O., Bannink, A. (2011). Dietary strategies to reducing N excretion from cattle: Implications for methane emissions. Current Opinion in Environmental Sustainability. Wageningen University, Wageningen -The Netherlands. 3(5), 414-422. https://10.1016/j.cosust.2011.07.008

Ellis-Lynn, J., Dijkstra, J., Kebreab, ER., Bannink, A., Odongo, N., Mcbride, BW., France, J. (2008). Aspects of rumen microbiology central to mechanistic modelling of methane production in cattle. The Journal of Agricultural Science, J Agri Sci. University of Guelph-Canada. 146(2), 213-233. https://doi.org/10.1017/S0021859608007752

Federación Colombiana de Ganaderos - FEDEGAN. (2018). Número de bovinos de acuerdo a la orientación de su producción. 004-Orientación del hato ganadero. Oficina de Planeación Fedegán, Colombia. https://www.fedegan.org.co/estadisticas/inventario-ganadero

Food and Agriculture Organization of the United Nations and Global Dairy Platform Inc. FAO. (2019). Climate Change and the Global Dairy Cattle Sector. FAO. Roma. https://bit.ly/36ANqRq

Firkins-Lynn, J., y Zhongtang, Y. (2015). Ruminant Nutrition Symposium: How to use data on the rumen microbiome to improve our understanding of ruminant nutrition1,2. Journal of Animal Science, Jour A Sci. The Ohio State University, Columbus-United States. 93(4), 1450-1470. https://doi.org/10.2527/jas.2014$\underline{8754}$

Gerber, PJ., Mottet, A., Opio, C., Teillard, F. (2015). Environmental impacts of beef production: Review of challenges and perspectives for durability. Meat Science, United Nations. Animal Production and Health Division, Food and Agriculture Organization of the United Nations. 109, 2-12.https://bit.ly/3omOSg6

Granja-Salcedo, Y., Ribeiro-Júnior, C., De-Jesus, R., Gomez-Insuasti, A., Rivera, A., Messana, J., Canesin, R., Berchielli, T. (2016). Effect of different levels of concentrate on ruminal microorganisms and rumen fermentation in Nellore steers. Archives of Animal Nutrition. Universidad Estadual Paulista, Faculdade de Ciências Agrárias e Veterinárias, Departamento de Zootecnia, São Paulo-Brasil. 70:1, 17-32. http://dx.doi.org/10.1080/1745039X.2015.1117562

Granja-Salcedo, Y., Ramirez-Uscategui, R., Machado E., Messana, J., Kishi, L., Berchielli, T. (2017) Studies on bacterial community composition are affected by the time and storage method of the rumen content. Universidad Estadual Paulista, Faculdade de Ciências Agrárias e Veterinárias, Departamento de Zootecnia, São Paulo-Brasil. PLoS ONE. 12(4): e0176701. https://bit.ly/33IKWyp

Haque, M. (2018). Dietary manipulation: A sustainable way to mitigate methane emissions from ruminants. Journal of Animal Science and Technology, Bangabandhu Sheikh Mujibur Rahman Science and Technology University. Gopalganj-Bangladesh. 60(1), 15. https://doi.org/10.1186/s40781-018-0175-7

Haro, A., Carro, M., De Evan, T., González, J. (2018). Protecting protein against ruminal degradation could contribute to reduced methane production. Journal of Animal Physiology and Animal Nutrition. Universidad Politécnica de Madrid, Ciudad Universitaria, Madrid, España 102(6), 1482-1487. 


\section{https://doi.org/10.1111/jpn.12973}

IPCC (Intergovernmental Panel on Climate Change). (2019). Summary for Policymakers. In: Climate Change and Land: an IPCC special report on climate change, desertification, land degradation, sustainable land management, food security, and greenhouse gas fluxes in terrestrial ecosystems, United States. https://www.ipcc.ch/srccl/chapter/summary-for-policymakers/

Ishino, Y., Komori K., Cann, I., Koga, Y. (1998). ANovel DNA Polymerase Family Found in Archaea. Journal of Bacteriology. Department of Molecular Biology, Biomolecular Engineering Research Institute (BERI). Osaka- Japan. 180(8), 2232-2236. https://doi.org/10.1128/JB.180.8.2232-2236.1998

Leonardi, C., Stevenson, M., Armentano, L. (2003). Effect of Two Levels of Crude Protein and Methionine Supplementation on Performance of Dairy Cows. Journal of Dairy Science. University of Wisconsin, Madison, United States. 86(12), 4033-4042. https://doi.org/10.3168/jds.S0022-0302(03)74014-4

Li, F., Guan Le, L. (2017). Metatranscriptomic Profiling Reveals Linkages between the Active Rumen Microbiome and Feed Efficiency in Beef Cattle. Applied and Environmental Microbiology, 83(9), e0006117, e00061-17. University of Alberta, Edmonton, Alberta, Canada. https://doi.org/10.1128/AEM.00061$\underline{17}$

Rufino-Almeida, M., Detmann, E., Gomes, DW., Dos-Reis, LS., Darlisson-Batista, E., Campos Valadares, S., Paulino, MF. (2016). Intake, digestibility and nitrogen utilization in cattle fed tropical forage and supplemented with protein in the rumen, abomasum, or both. Journal of Animal Science and Biotechnology. Universidade Federal de Viçosa, Minas Gerais, Brasil. 7(1), 11. https://doi.org/10.1186/s40104-016-0069-9

Salazar, DR., Simoes-Cortinhas, C., Esler-Freitas, J. (2008). Sincronismo energia- proteína: assimilação de nitrogênio e síntese de proteína microbiana em ruminantes. Pubvet, Universidade de São Paulo, Pirassununga-Brasil v.2, n.15.http://www.pubvet.com.br/material/Salazar200.pdf

Shabat- Bem, K., Goor, S., Faigenboim, AD., Durman T., Yaacoby, S., Berg, ME., A White, B., Shterzer, N., Itzhak, M. (2016). Specific microbiome-dependent mechanisms underlie the energy harvest efficiency of ruminants. The ISME Journal, Ben-Gurion University of the Negev, Beer-Sheva -Israel. 10(12), 29582972. https://doi.org/10.1038/ismej.2016.62

Tapio, I., Snelling, T., Strozzi, F., Wallace, J. (2017). The ruminal microbiome associated with methane emissions from ruminant livestock. Jokioinen -Finland. Journal of Animal Science and Biotechnology -J Animal Sci Biotechnol. Green Technology, Natural Resources Institute Jokioinen-Finland. v.8, n.1, p.111.

United States Department of Agriculture (USDA). (2020). Livestock and Poultry: World Markets and Trade. United States Department of Agriculture Foreign Agricultural Service: USDA. https://www.fas.usda.gov/data/livestock-and-poultry-world-markets-and-trade

Valle, ER., Henderson, G., Janssen, PH., Cox, F., T, WA., Mcallister, TA. (2015). Considerations in the use of fluorescence in situ hybridization (FISH) and confocal laser scanning microscopy to characterize rumen methanogens and define their spatial distributions. Canadian Journal of Microbiology, Lethbridge Research Centre, Agriculture and Agri-Food Canada. 61(6), 417-428. https://doi.org/10.1139/cjm-2014$\underline{0873}$ 
Vanegas, J., González, J., y Carro, M. (2017). Influence of protein fermentation and carbohydrate source on in vitro methane production. Journal of Animal Physiology and Animal Nutrition, Universidad Politecnica de Madrid, Madrid, Spain. 101(5), e288-e296. https://doi.org/10.1111/jpn.12604

Vandehaar, MJ., N. St-Pierre. (2006). Major Advances in Nutrition: Relevance to the Sustainability of the Dairy Industry. Journal of Dairy Science, Michigan State University 89(4), 1280-1291. https://doi.org/10.3168/jds.S0022-0302(06)72196-8

Van Sanne, G., Jan, D., André, B. (2019). Are dietary strategies to mitigate enteric methane emission equally effective across dairy cattle, beef cattle, and sheep?. Journal of Dairy Science, Wageningen University \& Research. Wageningen-Netherlands102(7), 6109-6130. https://doi.org/10.3168/jds.2018-15785

Van Soest, P. (1994). Nutritional ecology of the ruminant. 2. ed. Ithaca: New York State College of Agriculture and Life Sciences, Cornell University. 476p.

Wallace, J., Rooke, JA., Nest, M., Duthie, C., Hyslop, JJ., Ross, D., Waterhouse, A., Mick, W., Rainer, R. (2015). The rumen microbial metagenome associated with high methane production in cattle. BMC Genomics16(1), University of Aberdeen, Bucksburn, Aberdeen, UK. https://doi.org/10.1186/s12864$\underline{015-2032-0}$

Yang, C., Rooke, JA., Cabeza, I., Wallace, R. (2016). Nitrate and Inhibition of Ruminal Methanogenesis: Microbial Ecology, Obstacles, and Opportunities for Lowering Methane Emissions from Ruminant Livestock. Frontiers in Microbiology, 7. Chinese Academy of Agricultural Sciences, Nanning, China. https://doi.org/10.3389/fmicb.2016.00132 